\title{
Dependence of Exchange Bias Field on Thickness of Antiferromagnetic Layer in NiFe/IrMn Structures
}

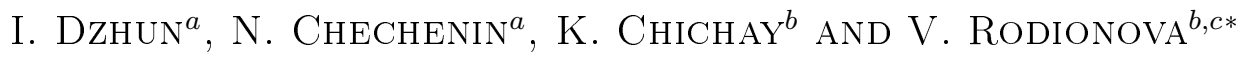 \\ ${ }^{a}$ Skobeltsyn Institute of Nuclear Physics, Lomonosov Moscow State University, \\ Leninskie gory 1(2), 119991 Moscow, Russia \\ ${ }^{b}$ Innovation Park and Institute of Physics and Technology, Immanuel Kant Baltic Federal University, \\ Nevskogo 14, 236041 Kaliningrad, Russia \\ ${ }^{c}$ National University of Science and Technology "MISIS", Leninskiy pr. 4, 119049 Moscow, Russia

\begin{abstract}
Magnetic properties of ferromagnetic/antiferromagnetic thin-films structures for spin-valve applications have been studied. Multilayer structures of $\mathrm{Ta} / \mathrm{Co} / \mathrm{IrMn} / \mathrm{Ta}$ and $\mathrm{Ta} / \mathrm{FeNi} / \mathrm{IrMn} / \mathrm{Ta}$ were deposited on Si substrate at room temperature by DC magnetron sputtering. Thickness of the antiferromagnetic layer changed from 10 to 50 nm. The coercive force was found to be non-monotonic function of the antiferromagnetic layer thickness. The exchange bias for 30-50 nm antiferromagnetic layers (73 Oe) is about 10 Oe larger than for 10-20 nm antiferromagnetic layers. Moreover, it was demonstrated that the alternative sequence of the deposition (antiferromagnetic layer on the top or below the ferromagnetic layer) leads to dramatic changes of structures magnetic properties.
\end{abstract}

DOI: 10.12693 /APhysPolA.127.555

PACS: $75.30 . \mathrm{Gw}, 77.55 .-\mathrm{g}$

\section{Introduction}

For further development of giant-magnetoresistancebased magnetic-field sensors, it is important to obtain exchange bias (EB) of a desirable magnitude together with other important functional properties of the structures such as corrosion and thermal stability [1]. The knowledge of the influence of the properties of the ferromagnetic $(\mathrm{F})$ and antiferromagnetic (AF) layer materials on the EB field magnitude is important for design of the devices with the required characteristics. But many effects concerning the EB are also of a fundamental interest. One of such effects is the influence of microstructure of F- and AF-layers on EB [2]. In this case the main factors are texture and grain size of AF layer and roughness of $\mathrm{F} / \mathrm{AF}$ interface. It was shown in $[3,4]$ that the order of $\mathrm{F}$ and $\mathrm{AF}$ layer deposition can define these factors and, consequently, affect the EB magnitude. The influence of the AF layer thickness on magnetic properties of samples has been studied in a number of works $[5,6]$. Besides the thickness, there are many other factors which influence the magnetic properties. This makes a prediction of the EB magnitude for different materials and methods of growth of the structure an ambiguous task, therefore only experimental studies can give a reliable set of data. In this work the influence of AF-layer thickness, as well as the sequence of the $\mathrm{F}$ and $\mathrm{AF}$ layers deposition, was experimentally studied for $\mathrm{NiFe} / \mathrm{IrMn}$ structures.

\section{Experimental techniques}

Experimental samples were obtained by DC magnetron sputtering in argon at the pressure of $3 \times 10^{-3}$ Torr. In order to saturate magnetic moment of the AF-layer,

\footnotetext{
* corresponding author; e-mail: rodionova@lnmm.ru
}

the magnetic field of 420 Oe was applied in plane of the substrate during the layer deposition. The advantage of this method is the absence of interdiffusion that takes place when the method of thermal annealing with subsequent field cooling is applied. There were obtained the TP (top pinned) bilayer structure, $\mathrm{Si} / \mathrm{Ta} 30 \mathrm{~nm} / \mathrm{NiFe} \quad 7 \quad \mathrm{~nm} / \mathrm{IrMn} \quad 15 \mathrm{~nm} / \mathrm{Ta}$ $30 \mathrm{~nm}$, and BP (bottom pinned) structure, $\mathrm{Si} / \mathrm{Ta} 30 \mathrm{~nm} / \mathrm{NiFe} 10 \mathrm{~nm} / \mathrm{IrMn} t_{\mathrm{AF}} / \mathrm{Ta} 30 \mathrm{~nm}$, with inverse order of F- and AF-layers and with $t_{\mathrm{AF}}=10$, 20, 30, 40, $50 \mathrm{~nm}$. The IrMn layers were deposited from IrMn target while $\mathrm{NiFe}$ layers were obtained by simultaneous co-deposition of $\mathrm{Fe}$ and $\mathrm{Ni}$ targets. The deposition rates for each layer were evaluated by measuring the thickness of the calibrating layers using the Rutherford backscattering.

The alloy composition was also checked by X-ray energy dispersive spectroscopy (XEDS) after the samples deposition and it was $\mathrm{Ni}_{75} \mathrm{Fe}_{25}$ and $\mathrm{Ir}_{30} \mathrm{Mn}_{70}$. In order to compare the magnetic properties and to find the optimal parameters of the structures, the samples, Si/Ta $30 \mathrm{~nm} / \mathrm{Co}$ (or NiFe) $7 \mathrm{~nm} / \mathrm{Ta} 30 \mathrm{~nm}$ and $\mathrm{Si} / \mathrm{Ta} 30 \mathrm{~nm} /$ Co $7 \mathrm{~nm} / \mathrm{IrMn} 15 \mathrm{~nm} / \mathrm{Ta} 30 \mathrm{~nm}$, were also deposited. These structures demonstrate so-called free F-layer, i.e. not pinned with $\mathrm{AF}$ by exchange interaction.

The magnetic properties of structures were investigated by vibrating sample magnetometry (VSM) and by angular distribution of the ferromagnetic resonance (FMR) field. Assuming the sample is aligned with an angle $\theta$ between the unidirectional EB field, which is parallel to the easy axis (EA) of the F-layer, and the FMR external magnetic field direction, then for $4 \pi M_{s} \gg H_{r}$ the resonance peak position is governed by intrinsic resonance field $H_{r 0}=(\omega / \gamma)^{2} /\left(4 \pi M_{s}\right)$ of F-layer, bidirectional uniaxial crystalline anisotropy field $H_{K}$ and uni- 
directional anisotropy or EB field $H_{\mathrm{EB}}$ :

$$
H_{r}=\frac{\omega^{2} / \gamma^{2}}{4 \pi M s}-H_{\mathrm{EB}} \cos \theta-H_{K} \cos 2 \theta .
$$

Since the EA direction in our samples is set by the direction of the field during the deposition, $\theta$ is, in fact, the angle between the DC magnetic FMR field direction and the direction of deposition field. Parameters $H_{r 0}$, $H_{\mathrm{EB}}$ and $H_{K}$ were calculated through theoretical fitting of FMR field distribution by Eq. (1).

The surface properties of the samples were examined by atomic force microscopy (AFM).

\section{Experimental results and discussion}

F-layers demonstrate different changes in the presence of AF-layer. Hysteresis loops, normalized to the maximum value of magnetic moment for each sample, are shown in Fig. 1. The results of magnetic properties investigation of Co $7 \mathrm{~nm} / \operatorname{IrMn} 15 \mathrm{~nm}, \mathrm{NiFe} 7 \mathrm{~nm} / \operatorname{IrMn} 15 \mathrm{~nm}$ structures and of free F-layers with $7 \mathrm{~nm}$ thickness are represented in Table.

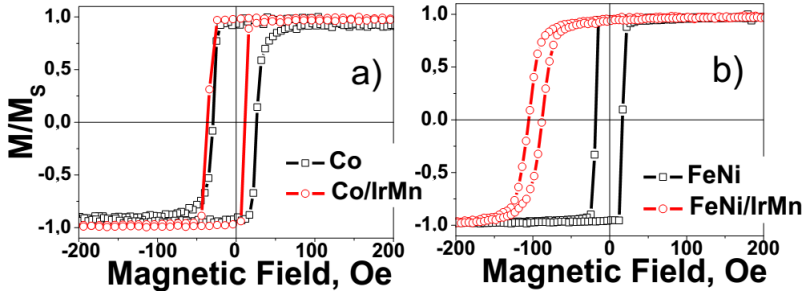

Fig. 1. Hysteresis loops for free and pinned by IrMn (a) Co and (b) NiFe layers.

TABLE

The results of investigations of magnetic properties of pinned and free F-layers.

\begin{tabular}{c|c|c|c|c|c}
\hline \hline Sample & $\begin{array}{c}H_{\mathrm{c}} \\
{[\mathrm{Oe}]}\end{array}$ & $\begin{array}{c}H_{\mathrm{EB}}[\mathrm{Oe}] \\
(\mathrm{VSM})\end{array}$ & $\begin{array}{c}H_{\mathrm{EB}}[\mathrm{Oe}] \\
(\mathrm{FMR})\end{array}$ & $\begin{array}{c}H_{\mathrm{ro}} \\
{[\mathrm{Oe}]}\end{array}$ & $\begin{array}{c}H_{K} \\
{[\mathrm{Oe}]}\end{array}$ \\
\hline Free Co & 28 & - & - & 596 & 65 \\
Free NiFe & 17.5 & - & - & 750 & 10 \\
$\mathrm{Co} / \mathrm{IrMn}$ & 24 & 12 & 14 & 533 & 134 \\
NiFe/IrMn & 9 & 95 & 85 & 747 & 15
\end{tabular}

EB magnitude in $\mathrm{NiFe} / \mathrm{IrMn}$ bilayer is much greater than in $\mathrm{Co} / \mathrm{IrMn}$ one that can be caused by higher coercivity of Co. Both free Co layer and Co/IrMn structure are characterized by high uniaxial anisotropy values. Low values of intrinsic resonance field indicate on high saturation magnetization. Thus NiFe F-layer material is more perspective for future application and it was chosen for further AF layer thickness dependence investigations.

The VSM curves for TP- and BP-structures are represented in Fig. 2a and b, respectively. The samples magnetization was normalized to its saturation value. At room temperature the TP-structure demonstrated EB of 85 Oe. The coercivity of this structure in the easy axis direction was 9 Oe that is lower than coercivity of free F-layer $(17.5 \mathrm{Oe})$. The TP-sample coercivity with the magnetic field applied in direction perpendicular to easy axis was significantly lower than that of free layer: 3 Oe in case of TP-structure and 29 Oe in case of free layer. The BP-structure showed the EB up to $121 \mathrm{Oe}$, much higher than that in TP-structure. In line with EB, a larger coercivity was observed in the BP samples, equal to 136 Oe along the easy axis and 72 Oe in perpendicular direction that is much higher than coercivity of TPstructure and the free $\mathrm{F}$ layer.

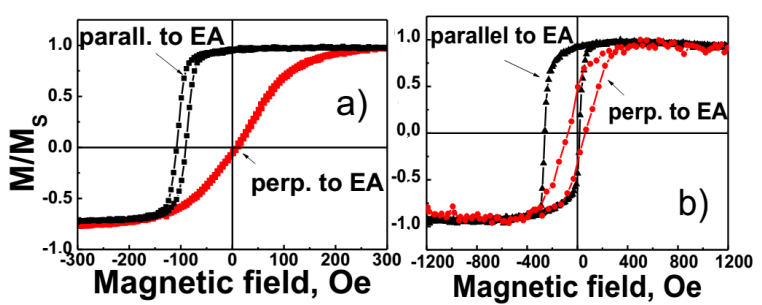

Fig. 2. Hysteresis loops for TP- and BP-structures: (a) NiFe $7 \mathrm{~nm} / \mathrm{IrMn} 15 \mathrm{~nm}$ and (b) IrMn $15 \mathrm{~nm} / \mathrm{NiFe}$ $7 \mathrm{~nm}$ for easy axis and perpendicular to easy axis are shown.

The AFM measurements revealed that BPstructures are characterized with smoother surface. The structures $\mathrm{Si} / \mathrm{Ta}(30 \mathrm{~nm}) / \mathrm{NiFe}(7 \mathrm{~nm})$ and $\mathrm{Si} / \mathrm{Ta}(30 \mathrm{~nm}) / \operatorname{IrMn}(15 \mathrm{~nm})$ were also examined by AFM in order to obtain information about underlying layer roughness that could be profitable to make some conclusions about interface roughness in TPand BP-structures. The surface of these structures was island-like with different roughness characteristics. The surface of $\mathrm{Si} / \mathrm{Ta}(30 \mathrm{~nm}) / \mathrm{NiFe}(7 \mathrm{~nm})$ structure is characterized with roughness height of $2 \mathrm{~nm}$, roughness diameter of $50 \mathrm{~nm}$ and concentration of $1001 / \mu \mathrm{m}^{2}$. In case of $\mathrm{Si} / \mathrm{Ta}(30 \mathrm{~nm}) / \operatorname{IrMn}(15 \mathrm{~nm})$ structure the interface roughness height was about $1 \mathrm{~nm}$, roughness diameter was over $100 \mathrm{~nm}$ and roughness density was $851 / \mu \mathrm{m}^{2}$.

The fact that EB magnitude is larger for BP- rather than for TP-structures is surprising because earlier [7] there was no EB observed for BP-structures with IrMn AF-layer. This difference is probably caused by the fact that the different methods of layer deposition and AFlayer saturation were used. It was shown [8] that the presence and magnitude of magnetic field applied during layer deposition affects on EB that is most probably caused by the changes in the F- and AF-layers textures. When the layer deposition is carried out without magnetic field the F-layer surface is quite smooth that makes the $\mathrm{F} / \mathrm{AF}$ interface in TP-structures also smooth. With appearance of magnetic field during the layer deposition the F-layer surface becomes island-like that leads to rough interface in TP-structures and correspondingly lowers the EB magnitude. In work [9] it was also shown that the surface of BP-structures is smoother than that of TP-structures. Our AFM investigations have also shown different roughness of IrMn and NiFe underlying layers that leads to different interface roughness in TP- 
and BP-structures. That finally results in a different magnetic properties such as EB and coercivity. The fact that the difference in roughness of underlying layers is not very large signalizes that roughness is only one of parameters that changes with inverse order of F- and AF-layers deposition. Other factors, like texture, grain size and AF-domain structure may also vary with change of $\mathrm{F}$ and AF deposition order. The influence of IrMn texture and grain size on EB is still not clear. In work [10] it was shown that strong (111) IrMn texture corresponds to higher EB. At the same time in [1] the inverse dependence of EB on (111) IrMn texture is reported. In work [11] it was shown that (111) IrMn texture affects EB more than grain size, while in work [3] it was shown that grain size plays a key role in difference of EB in TP and BP structures. The IrMn grain size difference in BP and TP can be a reason for a large coercivity enhancement in BPstructure, seen in Fig. 2 [12].

The FMR field angular distribution in the sample with $t_{\mathrm{AF}}=10 \mathrm{~nm}$ is shown in Fig. 3a. This distribution has a symmetric bell-like shape and experimental points are in a good agreement with theoretical fitting by Eq. (1). Figure 3b shows the angular dependence of EB for the same sample, it is typical for exchange-bias samples.

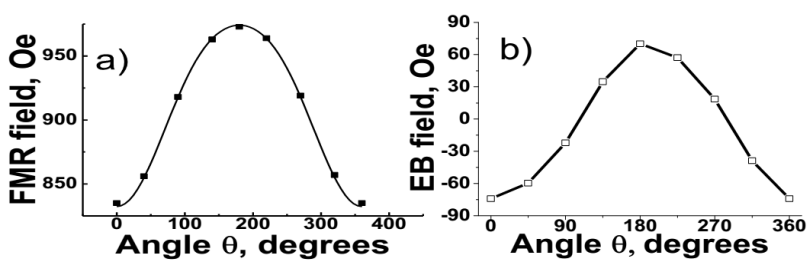

Fig. 3. (a) FMR field angular distribution. The solid line is a theoretical fit by Eq. (1); (b) the angular dependence of EB for structure NiFe $10 \mathrm{~nm} / \mathrm{IrMn} 10 \mathrm{~nm}$.

The dependence of the EB on AF layer thickness is represented in Fig. 4a. The EB is the same at $t_{\mathrm{AF}}=10$ and $20 \mathrm{~nm}$, then slightly increase at $t_{\mathrm{AF}}=30 \mathrm{~nm}$ and then again becomes thickness independent. There was also observed a slight coercivity enhancement at $t_{\mathrm{AF}}=$ $40 \mathrm{~nm}$ in both easy and hard axes directions (Fig. 4b).

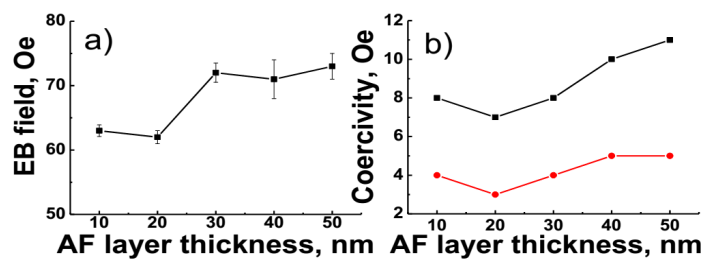

Fig. 4. AF layer thickness dependence of (a) EB field; (b) coercivity along easy axis (squares) and perpendicular to it (circles), for structure NiFe $10 \mathrm{~nm} / \mathrm{IrMn} t_{\mathrm{AF}}$.

Our EB values are comparable with those from bibliography. As examples, the values of $\mathrm{EB}$ for similar $\mathrm{NiFe} / \mathrm{IrMn}$ structures were reported, 60 Oe for $20-60 \mathrm{~nm}$ AF layer and $25 \mathrm{~nm} F$ layer [1], 45 Oe for $30 \mathrm{~nm} \mathrm{AF} \mathrm{layer}$ and $30 \mathrm{~nm} \mathrm{~F}$ layer [13]. This demonstrates that optimal combination of exchange bias and coercivity can be obtained by a proper choice of AF and F order and thickness.

\section{Conclusion}

Using angular dependence of FMR and VSM technique magnetic properties of $\mathrm{F} / \mathrm{AF}$ thin-films structures for spin-valve applications have been studied with variation of the AF layer thickness from 10 to $50 \mathrm{~nm}$. The $H_{\mathrm{C}}$ was found to be non-monotonic function of the AF layer thickness. The EB for $30-50 \mathrm{~nm}$ AF layers is about 10 Oe larger than for 10-20 nm AF layers. It was found that the alternative sequence of the deposition (AF layer on the top or below the $\mathrm{F}$ layer) leads to dramatic changes of the magnetic properties of bilayer structures. To achieve the maximum effect of the EB the optimal AF layer thicknesses were found to be $30-50 \mathrm{~nm}$.

\section{Acknowledgments}

The work was carried out with financial support in part from the Ministry of Education and Science of the Russian Federation in the frameworks of Increase Competitiveness Program of MISiS and of government assignment (No. 3.2582.2014/K); and from RFBR grant \# 14-3250871. The FMR study was performed at User Facilities Centre of Lomonosov MSU.

\section{References}

[1] A.J. Devasahayam, P.J. Sides, M.H. Kryder, J. Appl. Phys. 83, 7216 (1998).

[2] J. Nogues, I.K. Shuller, J. Magn. Magn. Mater. 192, 203 (1999).

[3] Jae-Chul Ro, Young-Snk Choi, Su-Jung Suh, HwackJoo Lee, IEEE Trans. Magn. 35, 3952 (1999).

[4] E.V. Khomenko, N.G. Chechenin, I.O. Dzhun, N.S. Perov, V.V. Samsonova, A.Yu. Goikhman, A.V. Zenkevich, Phys. Solid State 52, 1701 (2010).

[5] M. Ali, C.H. Marrows, M. Al-Jawad, B.J. Hickey, A. Misra, U. Nowak, K.D. Usadel, Phys. Rev. B 68, 214420 (2003).

[6] Feng Xu, Zhiqin Liao, Qijun Huang, N.N. Phuoc, Chong Kim Ong, Shandong Li, IEEE Trans. Magn. 47, 10 (2011).

[7] E.V. Khomenko, N.G. Chechenin, A.Yu. Goikhman, A.V. Zenkevich, JETP Lett. 88, 602 (2008).

[8] I.O. Dzhun, S.A. Dushenko, N.G. Chechenin, E.A. Konstantinova, J. Phys. Conf. Ser. 303, 012104 (2011).

[9] V. Kunser, M. Valeanu, G. Schinteie, G. Filoti, I. Mustata, C.P. Lungy, A. Anghel, H. Chriac, R. Vladoiu, J. Bartolome, J. Magn. Magn. Mater. 320, 226 (2008).

[10] K. Hoshino, R. Nakatani, H. Hoshiya, Y. Sugita, S. Tsunashima, Jpn. J. Appl. Phys. 35, 607 (1996).

[11] J. van Driel, F.R. de Boer, K.-M.H. Lenssen, R. Coehoorn, J. Appl. Phys. 88, 975 (2000).

[12] H.S. Jung, W.D. Doyle, H. Fujiwara, J.E. Wittig, J.F. Al-Sharab, J. Bentley, N.D. Evans, J. Appl. Phys. 91, 6899 (2002).

[13] J. van Driel, R. Coehoorn, K.-M.H. Lenssen, A.E.T. Kuiper, F.R. de Boer, J. Appl. Phys. 85, 5522 (1999). 